\title{
Bites before and after bedtime can carry a high risk of human malaria infection
}

\author{
Masabho P. Milali1 ${ }^{1,2}$, Maggy T. Sikulu-Lord ${ }^{1,3}$ and Nicodem J. Govella ${ }^{1 *}$
}

\begin{abstract}
Background: Understanding biting distribution of potentially infectious (parous) mosquitoes at various hours of the night would be useful in establishing the likely impact of bed nets on malaria transmission. Bed nets are highly effective at preventing biting by older malaria vectors, which occurs when most people are in bed. However, this behaviour is likely to vary across ecological settings and among mosquito populations.

Methods: Field experiments were conducted in Minepa village within Kilombero Valley. Two outdoor catching stations located approximately $50 \mathrm{~m}$ from each other were established for mosquito collection. On each experimental night, mosquitoes were collected using human landing catch (HLC) by a single adult male at each station from 18:00 to $07: 00 \mathrm{~h}$. To compare the distribution of mosquito biting and the composition of their age structure, mosquitoes were sorted and recorded according to the hour they were collected. A sub-sample of Anopheles arabiensis was dissected to determine their parity status. Insectary-reared An. arabiensis within the semi-field system (SFS) with known age were also released in the SFS $(10 \mathrm{~m} \times 20 \mathrm{~m})$ and recaptured hourly using HLC to determine the effect of parity on biting distribution.
\end{abstract}

Results: Overall, there was no statistical association between the parity status and the biting time of An. arabiensis either in the field or in the SFS $(P \geq 0.05)$. The wild and insectary-reared An. arabiensis were observed to exhibit different hourly biting patterns.

Conclusion: The study has shown that mosquito biting time phenotype is not influenced by their parity status. These findings imply that the risk of human exposure to potentially infectious bites is equally distributed throughout the night, thus supplementary measures to protect people against bites in evening and morning are desirable.

Keywords: Malaria, Mosquito, Parity, Biting time, Transmission, Mosquito age, Distribution

\section{Background}

Historically, human malaria infections in sub-Saharan Africa occur mainly during late hours of the night. This period coincides with the peak biting behaviour of the primary malaria vectors: Anopheles gambiae sensu lato and Anopheles funestus [1,2]. The risk of human infection depends mainly on two main factors: the human biting rate (the frequency at which a human is exposed to mosquito bites) and the proportion of the biting mosquitoes that are infectious [3, 4]. Only anopheline

\footnotetext{
*Correspondence: govella@ihi.or.tz

1 Ifakara Health Institute, Environmental Health and Ecological Sciences Thematic Group, Coordination Office, PO Box 78373, Kiko Avenue, Mikocheni, Dar es Salaam, United Republic of Tanzania

Full list of author information is available at the end of the article
}

mosquitoes that are at least 10 days old can be infectious [5] because of the lengthy period required by the parasite to develop inside the mosquito, which is described as the extrinsic incubation period. While young nulliparous Anopheles never become infectious, the parous female may do so. Consequently, the proportion of mosquitoes that are infectious is proportional to the age of that mosquito populations [6, 7]. Therefore, the risk of infection at any given time of the night is influenced by the biting behaviour of the parous female mosquitoes $[6,7]$ : those that have previously had a blood meal and laid eggs [8-11].

Previous studies on An. gambiae in Sierra Leone, Anopheles punctulatus in Papua New Guinea [12] and Anopheles darlingi in Brazil [9] have provided evidence to indicate 
that parous Anopheles prefer to feed later in the night than the nulliparous population. The proportion of An. gambiae population that were parous in Tanzania was also slightly higher during late night hours (22:00-02:00) than earlier in the night (18:00-22:00) [13]. This overlap between the peak biting time of parous mosquitoes and the sleeping pattern of humans could explain why insecticidal-treated nets (ITNs) have been effective in interrupting human malaria infection across sub-Saharan Africa [14-19].

More recently, it has been reported that a substantial change in species composition of malaria vectors $[20,21]$ and a shift in biting time [21-27] is associated with the widespread use of ITNs across Africa. For instance, in Kilombero Valley in Tanzania, An. gambiae sensu stricto, which historically has been the dominant malariatransmitting species, has been virtually eliminated [21, 28]. It has also been reported that the biting behaviour of mosquitoes is increasingly occurring before bedtime and outdoors $[21,29]$. If the shift to bite before bedtime coincides with the increase in the proportion of parous mosquitoes, then the risk of malaria infection will be predictably relatively higher in this specific time window of the night compared to other time points.

Despite high coverage with ITNs, the villages around the Kilombero River still experience high malaria transmission rates $[28,30]$. However, the influence of age of the main malaria vector species on their biting behaviour among these villages remains unknown. It is evaluated here for the first time.

\section{Methods}

\section{Study site: Minepa village in southeastern Tanzania}

The field study was conducted in Minepa village (S $08^{\circ} 16.4974^{\prime}$; E $036^{\circ} 40.7640^{\prime}$ ) within the Kilombero River valley in the Ulanga district of southeastern Tanzania [31] where malaria transmission remains high despite high coverage with ITNs. Most people in this village are subsistence farmers. The annual rainfall is between 1200 and $1800 \mathrm{~mm}$, and the daily temperature is between 20 and $33{ }^{\circ} \mathrm{C}$ [32]. Members of the An. gambiae s.l. (An. gambiae s.s., An. arabiensis) and An. funestus are the primary malaria mosquitoes. However, An. arabiensis and An. funestus are currently the dominant species [21, 28] because long and widespread use of ITNs [33, 34] has virtually crashed the population of An. gambiae s.s. [21, 28]. Recent observations indicates that both $A n$. arabiensis and $A n$. funestus in this valley present active biting behaviour even before bedtime (18:00-22:00) [21], particularly when most locals are still outdoors [29].

\section{Experimental design}

\section{Field sampling and processing}

This experiment was conducted for two rounds each comprising a total of ten sampling nights. The first round was conducted towards the end of the rainy season between 21 and 30 April, 2016, while the second round was during the dry season 23 August to 1 September, 2016. Two outdoor catching stations, each approximately 5 m outside houses within the sampling area, were randomly chosen and established for mosquito collection. Standard randomization techniques were used: from the centre of the village two directions to work through the village were chosen by spinning a pen on a flat surface and the tenth house from each direction was chosen. Two volunteers out of four were randomly chosen and each randomly assigned to each catching station. Once assigned to a particular station, a volunteer was allowed to choose a counterpart to form a pair so that they can make a night shift with one start collection of mosquitoes from the first half of the night (18:00-24:00) and another finishing the second half of the night (24:00-07:00). Each pair remained at a particular station for a period of ten consecutive nights of sampling while alternating in night shift after each experimental night. In the second round, the two pairs exchanged houses and sampling continued for another ten consecutive nights in similar fashion as above. Mosquito collection was done by human landing catch (HLC), where a single male adult volunteer collected mosquitoes that landed on his exposed legs with a mouth aspirator as previously described $[35,36]$. Mosquito collection was conducted for $45 \mathrm{~min}$ per hour, from 18:00 to 07:00, allowing a 15-min break for rest and refreshment. To compare distribution of mosquito biting behaviour and age composition per time point, hourly collections were placed in separate labelled paper cups corresponding to capturing time.

Each morning, with the aid of a stereo-microscope, all catches were sorted and morphologically identified in the field. Only mosquitoes identified as An. gambiae complex or An. funestus [1, 37] were considered for follow up. All other mosquito species were identified, recorded and then discarded. Individual mosquitoes were dissected and identified as either pre-gravid, nulliparous or parous, as previously demonstrated [38]. These mosquitoes were then individually preserved in $1.5 \mathrm{ml}$ Eppendorf tubes containing desiccated silica gel for subsequent testing using polymerase chain reaction (PCR) assay [39] which determines sibling species identity. The enzyme linked immunosorbent assay (ELISA) was applied to test for the presence of a circumsporozoite protein in heads and thoraces of these mosquitoes [40, 41]. The heads and thoraces were heated in ELISA lysate at $100{ }^{\circ} \mathrm{C}$ for $10 \mathrm{~min}$ to gate away from false positive ELISA [42].

\section{Mark release recapture experiments in the semi- field system}

Insectary-reared female An. arabiensis collected from wild larvae in Lupiro village within the Kilombero Valley 
were used. Mosquitoes were reared in an insectary built within the Ifakara Health Institute's large semi-field system (SFS), measuring $21 \times 9.1 \times 7.1 \mathrm{~m}$ located at Kining'ina village (8.11417 S, 36.67484 E). Details of the design of the SFS can be seen elsewhere [43, 44]. The larvae from the field were transferred to a basin containing clean tap water using micropipette to discriminate predators. The basins were 12-1 volume each with $300 \mathrm{ml}$ of water. The basins containing larvae were covered with netting material and placed in racks established in the insectary within the SFS. The larvae were fed on Tetramin fish food (Tetra, Melle, Germany); temperature and humidity were not controlled during larvae rearing to mimic field conditions. Pupae were aspirated from the basins using micropipette and placed into small bowls (10 cm diameter) containing water and then transferred inside cages measuring $36 \times 39 \mathrm{~cm}$, allowing adult mosquitoes to emerge. The newly emerged wild adult female An. gambiae s.l. were maintained on $10 \%$ glucose solution until they were 3-5 days old when they were blood fed using arm feeding. Standard operating procedure (SOP) of arm feeding was followed, where well-trained technicians fed mosquitoes inside cages for $15 \mathrm{~min}$. Prior to feeding, technicians were screened for malaria by rapid diagnostic test (mRDT) (MAL-Pf ${ }^{\circledR}$, ICT Diagnostics, Cape Town, South Africa, which detects histidine-rich protein II) available in the laboratory. Only malaria freetechnicians were allowed to enter and feed mosquitoes in the insectary. Before feeding, technicians put on gloves to avoid mosquito bites around the fingers. This procedure received ethical approval following the fact that An. arabiensis strain in this setting had repeatedly failed to adapt to feeding upon animals, or membrane feeding from previous trials. It was approved based on the fact that mosquitoes which are reared in the insectary within the screened enclosures (SFS) are pathogen-free so that technicians and/or volunteers are not exposed to the risk of contracting diseases.

The fully fed female An. gambiae s.l. were individually placed into a correspondingly separate, labelled small cage $(15 \times 17 \mathrm{~cm})$ with unique mosquito identification code (ID). Inside each small cage, a petri dish containing wet cotton lined on top with filter paper was provided, allowing mosquitoes to oviposit eggs. After oviposition, each individual was killed and stored in a correspondingly labelled 1.5-ml Eppendorf tube with desiccated silica gel and taken to central laboratory of the Ifakara Health Institute for confirmation of sibling species identification by PCR [39]. The newly emerged adult female F1 generation confirmed to be An. arabiensis only were placed into two separate cages each measuring $36 \times 39 \mathrm{~cm}$. In one cage, the young nulliparous group was maintained on $10 \%$ glucose solution alone, while in the second cage young mosquitoes were blood fed and allowed to lay eggs three times. Three to five days old nulliparous females and parous mosquitoes that had undergone three feeding cycles (at least 10 days old) were used. To discriminate parous from the nulliparous age group, one night prior to the release-recapture experiment, 200 nulliparous and 200 parous mosquitoes were placed in two different cages and were maintained on a mixture of $10 \%$ glucose with $2 \mathrm{~g} / \mathrm{l}$ of either rhodamine B or synthetic blue food colour. The rhodamine B or blue food colour was assigned to either nulliparous or parous in randomized fashion using the lottery method. This randomization of markers between the two age groups was done after each experimental night. Although rhodamine $\mathrm{B}$ is a common biomarker for insects and was recently tested against sand flies [45], the use of synthetic food colour for marking mosquitoes is relatively rarely applied [46] compared to fluorescent dust dye [47, 48]. Nevertheless, this marking technique proved successful in this study (Fig. 1). The use of sugar-feeding dyes was preferred over fluorescent dust dye $[47,48]$ so that the

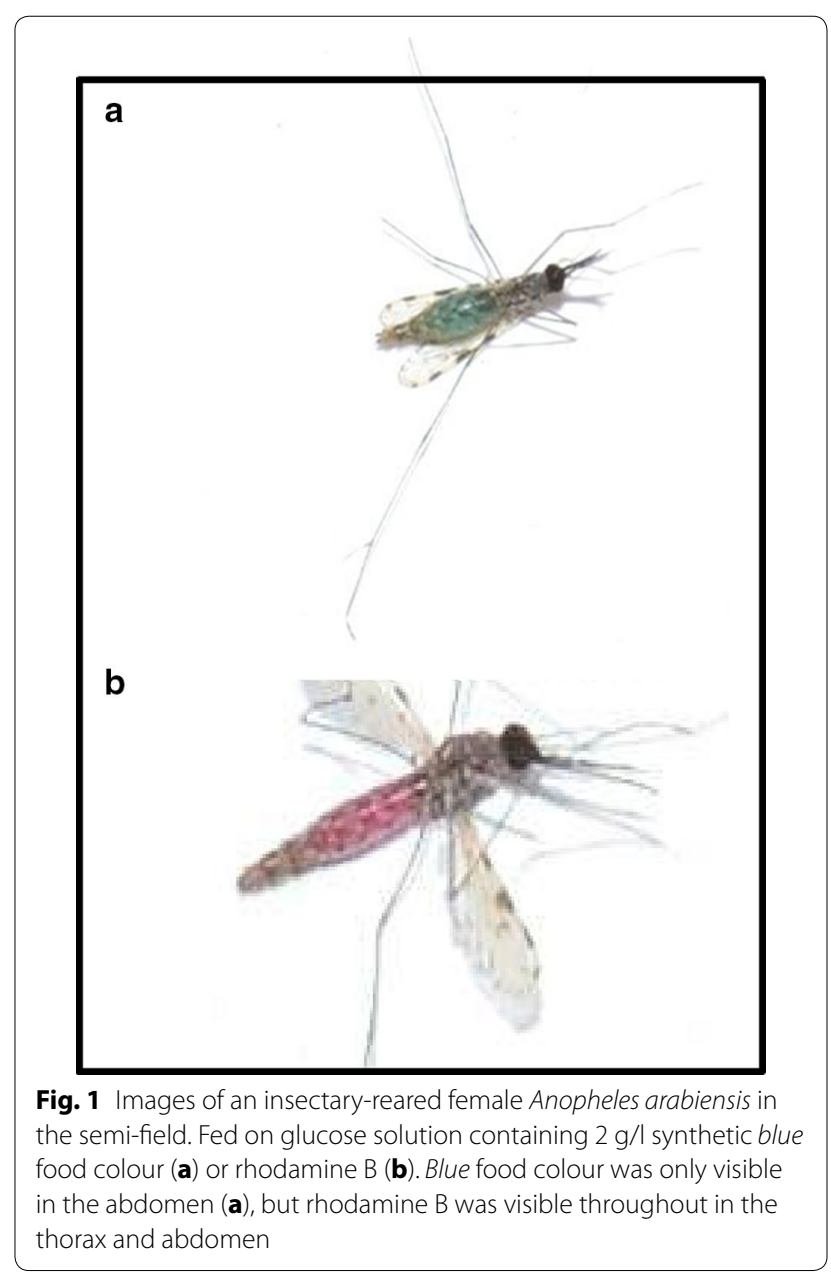


two age groups could be distinguished from each other. Unlike sugar-feeding markers, fluorescent dust dye may contaminate the aspirator during recapturing and make it difficult to discriminate between the two groups. Even more importantly, these sugar-feed markers, particularly the rhodamine $\mathrm{B}$, have been demonstrated not to affect longevity of the insects [45]. Mosquitoes were starved for at least $20 \mathrm{~min}$ before they were released. Mosquitoes were transferred and released in a separate chamber of the SFS containing natural vegetation, planted food crops and a small, thatched, mud-walled house designed to mimic the natural habitat of these mosquito species [44]. Mosquitoes were released from the centre of a chamber measuring $9.6 \times 9.6 \mathrm{~m}$ at 17:00 by pulling strings held to mosquito netting cage. Both parous and nulliparous mosquitoes were released at the same time. A single adult staff was introduced into the chamber at 18:00 to perform HLCs from 18:00 to 07:00. Mosquitoes were recaptured at intervals of $1 \mathrm{~h}$ and placed in paper cups with a label corresponding to the time they were recaptured. Similar to field trials, recapturing was conducted for $45 \mathrm{~min}$ per hour with a 15-min break. This experiment was conducted for ten consecutive nights. HLCs were performed by two research staff alternating after each experimental night. Because disease-free, insectary-reared mosquitoes were used, no prophylaxis was given to the mosquito catchers [49]. Not all mosquitoes that were released per experimental night were successfully recaptured by HLCs. After each experimental night, in the morning two technicians carried out a thorough search for $30 \mathrm{~min}$ in the vegetation, hut, walls, and roof within the SFS and all mosquitoes that were found resting were collected, killed and discarded. Otherwise this would have affected the results, especially for the next experimental nights.

\section{Data analysis}

Descriptive summary, tables and graphical analysis were used to examine the biting distribution across different times of the night for both wild An. arabiensis and An. funestus collected and insectary-reared $A n$. arabiensis. Generalized linear mixed models (GLMM), using the $\mathrm{R}$ open source statistical software (version $\mathrm{Rx} 64$ 2.15.2) augmented with the lme4 package, was applied to assess whether biting time phenotype was influenced or not by the parity status of mosquitoes and whether the proportion of parous varied between nights of sampling. The analysis of whether the proportion of parous recaptured varied between nights of sampling was only conducted with the SFS experiment. Very few pre-gravid mosquitoes were caught, and therefore they were combined with the nulliparous population and analysed as one group (nulliparous). Thus, the results of dissections were expressed as either nulliparous or parous. To test for the effect of parity on biting distribution across various times of the night for the field data, proportion of parous biting was treated as response variable with hour of the night first fit as a continuous variable and sampling night, rounds of collection and volunteers nested within station of collection treated as random effects. This allowed detection of whether there was any significant difference. This was followed by slight modification of the model where hour of the night at this stage was fit as fixed effect with random effects remaining the same as above, and model was run without an intercept. This was done so that absolute proportional of parous biting could be compared between each hour and also allow for plotting of graph fitted with $95 \%$ confidence interval. For the An. arabiensis reared in the SFS, testing the effect of time on parous biting distribution, the night of sampling and volunteer were treated as random effects with hour of the night as fixed effect and proportion of parous biting as response. Testing whether the proportion of parous biting varies over nights of experiment in the SFS, sampling nights were treated as fixed effect with volunteers as random effect and proportion of parous as response variable. It has been reported that differential attractiveness by mosquitoes to people does occur [5052]. This could result in sampling variations over a certain time of the night or between sampling night, especially when mosquito capturing is performed by more than one person. It was also hypothesized that differential attractiveness by parous and nulliparous mosquitoes to people may exist, so volunteers were controlled in the model by treating them as random effects. Binomial distribution was used and the model fit were then separately plotted into graphical presentation. Very few An. funestus was caught and when tested with GLMM, spurious model fit was produced. Therefore, their number was considered too low to justify any robust statistical test, and the results associated with An. funestus were only reported descriptively.

\section{Results}

A total of 5836 mosquitoes were caught over 20 nights of field collections. The catch included: 1710 (29.3\%) An. gambiae s.l.; 211 (3.6\%) An. funestus; 172 (2.9\%) Anopheles coustani; 11 (0.2\%) Anopheles ziemanni; 122 (2.1\%) Anopheles pharoensis; 3610 (62\%) Culex spp. (Table 1). A total of 1461 An. gambiae s.l. were successfully dissected. Of these, $63.2 \%(n=924)$ were parous, $30.8 \%(n=450)$ nulliparous and $6.0 \%(\mathrm{n}=87)$ pre-gravid. In the case of An. funestus, 200 specimens were successfully dissected. Of these, $66 \%(\mathrm{n}=132)$ were parous, $32.5 \%(\mathrm{n}=65)$ were nulliparous and $1.5 \%(\mathrm{n}=3)$ pre-gravid (Table 1$)$. Overall, there were relatively more parous than nulliparous mosquitoes, a probable indicator of fewer mosquitoes emerging towards the end of the rainy season and during the dry season. 
Table 1 Mosquito species, numbers collected and parity dissections of Anopheles gambiae sensu lato and Anopheles funestus from two rounds (21-30 April and 23 August-1 September, 2016) of data collection in Minepa Village, Kilombero Valley

\begin{tabular}{|c|c|c|c|c|c|c|c|}
\hline Collection rounds & Total catch & Mean catch per night & Total dissected & Parous & Nulliparous & Pregravid & Parous (\%) \\
\hline \multicolumn{8}{|l|}{ Round 1} \\
\hline Anopheles gambiae s.l. & 724 & 72.4 & 631 & 371 & 187 & 73 & 58.8 \\
\hline Anopheles funestus s.l. & 56 & 5.6 & 51 & 32 & 17 & 2 & 62.7 \\
\hline Anopheles coustani & 13 & 1.3 & N/A & N/A & N/A & N/A & N/A \\
\hline Anopheles ziemanni & 5 & 0.5 & N/A & N/A & N/A & N/A & N/A \\
\hline Anopheles pharoensis & 9 & 0.9 & N/A & N/A & N/A & N/A & N/A \\
\hline Culex spp. & 2301 & 230.1 & N/A & N/A & N/A & N/A & N/A \\
\hline \multicolumn{8}{|l|}{ Round 2} \\
\hline Anopheles gambiae s.l. & 986 & 98.6 & 830 & 553 & 263 & 14 & 66.6 \\
\hline Anopheles funestus s.l. & 155 & 15.5 & 149 & 100 & 48 & 1 & 67.1 \\
\hline Anopheles coustani & 159 & 15.9 & N/A & N/A & N/A & N/A & N/A \\
\hline Anopheles ziemanni & 6 & 0.6 & N/A & N/A & N/A & N/A & N/A \\
\hline Anopheles pharoensis & 113 & 11.3 & $\mathrm{~N} / \mathrm{A}$ & N/A & N/A & N/A & N/A \\
\hline Culex spp. & 1309 & 130.9 & N/A & N/A & N/A & N/A & N/A \\
\hline
\end{tabular}

Of the 4000 insectary-reared An. arabiensis within the SFS and released in the SFS, 1945 were recaptured. Of these, $69.4 \%(n=1349)$ were parous and $30.6 \%(n=596)$ were nulliparous. Of 1152 An. gambiae s.l. analysed by PCR, 96\% ( $\mathrm{n}=1106)$ specimens were successfully amplified and all were identified as An. arabiensis. All identified $A n$. arabiensis tested sporozoite negative. Contrary to that, two individuals (An. funestus s.s.) out of 181 from An. funestus s.l. were found sporozoite positive, with one biting between 21:00 and 22:00 and the other between 01:00 and 02:00. This implies that $A n$. funestus s.s. might be more susceptible to infection than An. arabiensis. The An. funestus group was composed of An. funestus s.s $86 \%$ $(\mathrm{n}=129)$, Anopheles leesoni 7\% $(\mathrm{n}=11)$, Anopheles rivulorum 4\% $(\mathrm{n}=6)$, and Anopheles parensis 3\% $(\mathrm{n}=4)$ of 150 successfully amplified specimens. Therefore, the field results presented here with respect to $A n$. gambiae s.l. and $A n$. funestus s.l. effectively reflect $A n$. arabiensis and An. funestus s.s.

The two primary malaria vectors in the area, $A n$. arabiensis and An. funestus, were observed to exhibit different biting activity over the course of the night. While the peak biting activity of $A n$. arabiensis appeared to have started as early as between 20:00 and 21:00 and thereafter gradually decreased; An. funestus exhibited nocturnal biting behaviour with additional pronounced peak biting behaviour observed in the early morning (Fig. 2). Interestingly, the wild and the insectary-reared $A n$. arabiensis within the SFS were also observed to exhibit different biting tendencies. Over $75 \%$ of all bites by the SFS-reared $A n$. arabiensis occurred during the first $2 \mathrm{~h}$ of the early evening but drastically dropped for the rest of the night (Fig. 3).

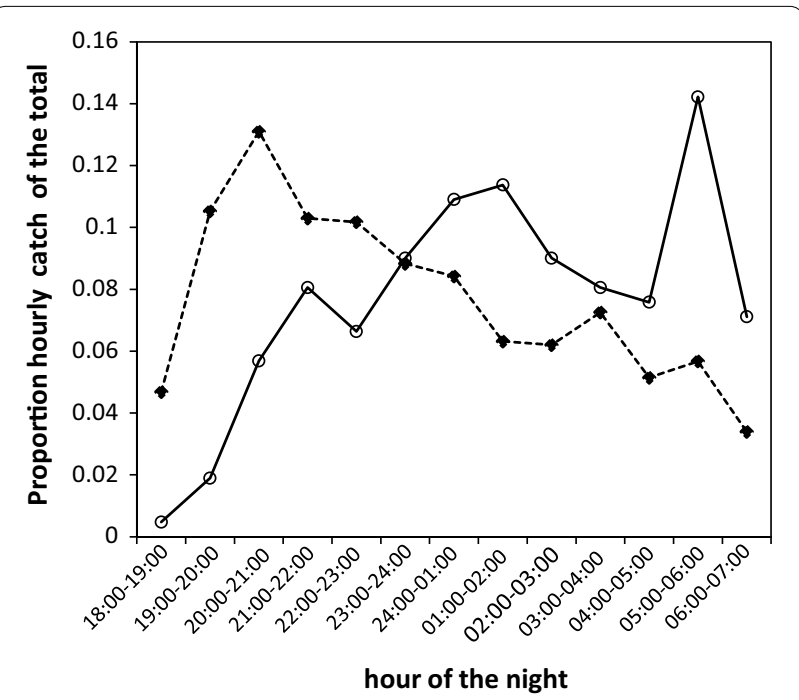

Fig. 2 Distribution of biting times for wild Anopheles arabiensis and Anopheles funestus in Kilombero Valley, Tanzania. The dashed line represents Anopheles arabiensis and the continuous line represents Anopheles funestus

Table 2 summarizes hour by hour numbers of wild female Anopheles arabiensis and An. funestus caught and numbers of parous, nulliparous and pre-gravid. Generally, the numbers of parous biting mosquitoes appeared to be consistently higher relative to nulliparous (nulliparous and pre-gravid combined) across different times of the night, with the exception of the first hour of the evening (18:00-19:00), which was dominated by nulliparous mosquitoes for An. arabiensis. This observation is supported by the statistical test, which shows the lack 


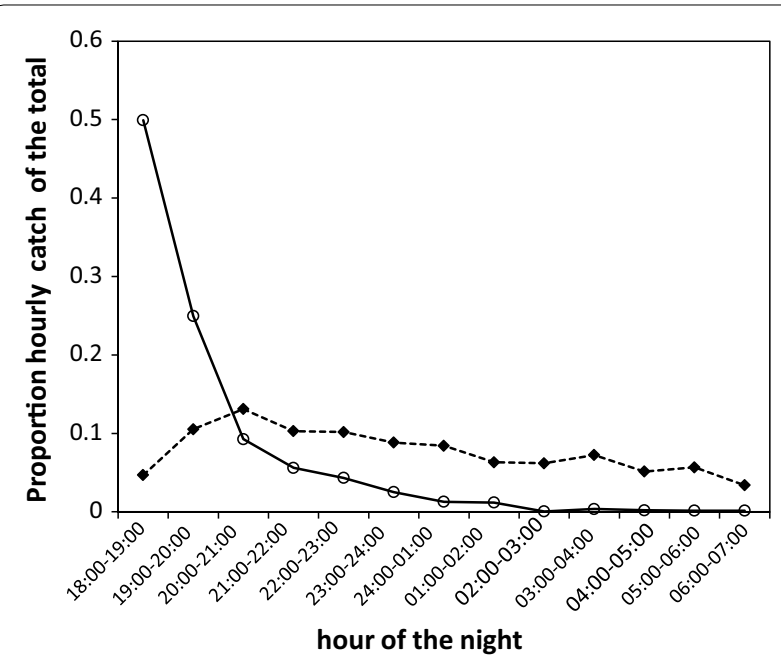

Fig. 3 Biting activity of wild Anopheles arabiensis in the field compared with insectary-reared Anopheles arabiensis in the semi-field system. The dashed line represents the proportion of An. arabiensis that were captured biting at each hour of the night in the field and continuous line represents $A n$. arabiensis that were recaptured biting at each hour of the night in the semi-field system

of detectably statistical association between the parity status and the biting time of wild An. arabiensis from 19:00 throughout the night. The only statistical difference was detected when the catches between 18:00 and 19:00 were included in the model, where the proportion of parous catch was significantly less $(\mathrm{z}=2.0, \mathrm{P}=0.045)$ (Fig. 4a). Although the numbers of An. funestus that were collected and dissected were too low to allow statistical test, proportion of parous biting from 20:00 appeared to be consistently $\geq 50 \%$ throughout the night (Table 2; Fig. 5). Similar to wild An. arabiensis, no apparent association was observed between biting time preference and parity status over the course of the night among the insectary-reared, released and recaptured An. arabiensis in the SFS. Only the biting activity between 04:00 and 05:00 appeared to be dominated by the nulliparous group (Fig. 4b).

As shown in Fig. 6, the parous rate of insectary-reared, released and recaptured An. arabiensis within the SFS was consistently $\geq 50 \%$ throughout the ten sampling nights, with some fluctuation between nights. Statistical analysis indicated no evidence of significant variation in parity rates between all ten nights (Fig. 6).

\section{Discussion}

A thorough understanding the biting behaviour of malaria vectors plays a crucial role in their control. Both An. funestus and An. arabiensis were collected, but the numbers of $A n$. funestus were too sparse to be able to detect the existence of any statistical difference in their age structure distribution. This discussion will therefore focus mainly on $A n$. arabiensis, the major vector of malaria in the Kilombero Valley, Tanzania, [21, 28, 29] and in other locations in sub-Saharan Africa [20, 24, 26, $27,53]$. The main objective was to assess whether there was an association between parity status of mosquitoes and their biting time in an area with widespread use of ITNs. Overall, the results indicate that parity status did not influence the biting time behaviour of $A n$. arabiensis either under a full-field or a semi-field setting. A relatively higher proportion of parous wild $A n$. arabiensis were observed to bite during the early hours of the

Table 2 Hour-by-hour numbers of wild female parous and nulliparous (nulliparous and pre-gravid combined) of Anopheles arabiensis and Anopheles funestus

\begin{tabular}{|c|c|c|c|c|c|c|}
\hline \multirow[t]{2}{*}{ Hour } & \multicolumn{3}{|c|}{ Anopheles arabiensis } & \multicolumn{3}{|c|}{ Anopheles funestus } \\
\hline & Parous & Nulliparous & Parous (\%) & Parous & Nulliparous & Parous (\%) \\
\hline $18: 00-19: 00$ & 21 & 51 & 29.2 & 0 & 2 & 0 \\
\hline 19:00-20:00 & 87 & 74 & 54.0 & 1 & 2 & 33.3 \\
\hline 20:0-21:00 & 129 & 57 & 69.4 & 10 & 4 & 71.4 \\
\hline $21: 0-22: 00$ & 97 & 42 & 69.8 & 12 & 4 & 75 \\
\hline $22: 0-23: 00$ & 105 & 42 & 71.4 & 5 & 5 & 50 \\
\hline $23: 0-24: 00$ & 85 & 38 & 69.1 & 12 & 6 & 66.7 \\
\hline $24: 0-01: 00$ & 66 & 52 & 55.9 & 15 & 9 & 62.5 \\
\hline 01:0-02:00 & 67 & 29 & 69.8 & 13 & 8 & 61.9 \\
\hline 02:0-03:00 & 57 & 28 & 67.1 & 12 & 5 & 70.6 \\
\hline 03:0-04:00 & 72 & 42 & 63.2 & 12 & 3 & 80 \\
\hline 04:0-05:00 & 56 & 25 & 69.1 & 11 & 4 & 73.3 \\
\hline 05:0-06:00 & 57 & 36 & 61.3 & 17 & 11 & 65.4 \\
\hline 06:0-07:00 & 25 & 19 & 56.8 & 12 & 5 & 70.6 \\
\hline
\end{tabular}




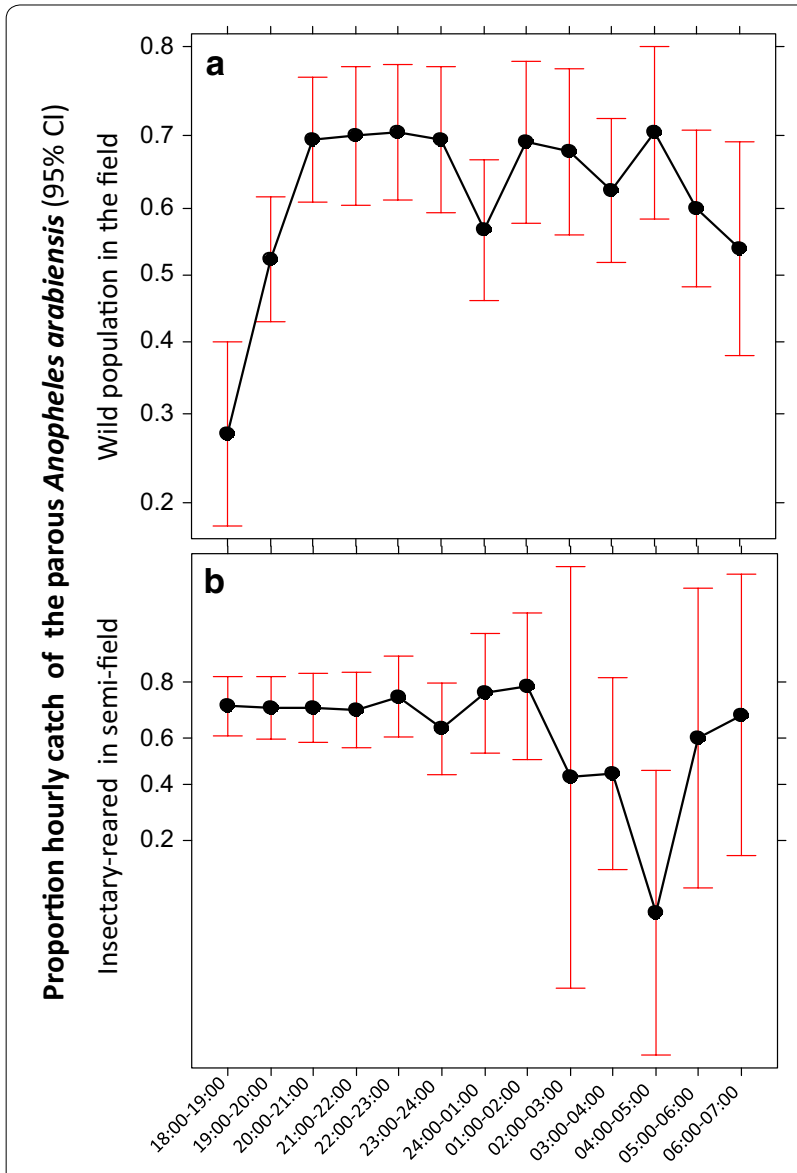

Fig. 4 The proportion of parous Anopheles arabiensis that were sampled biting across different hours of the night. a Represents parous rate that were captured in the field; $\mathbf{b}$ represents those that were released and recaptured in the semi-field system. Data points represent absolute proportion of parous biting at each hour and Bars represent the $95 \%$ confidence interval. X axis represents hour of the night

evening but this proportion was not significantly different from other time periods of the night. These findings differ from previous reports on An. gambiae in Sierra Leone, An. punctulatus in Papua New Guinea and $A n$. darling in Brazil, where the biting activity of the parous population predominantly peaked during bedtime, while the nulliparous population preferred to bite prior to or after bedtime [9, 12]. While local population variations in biting time behaviour with respect to mosquito age could possibly describe this difference, variation in the analytical approach may also matter. In previous studies, the proportion of parous was obtained through aggregating their numbers captured in ordered time intervals (e.g., 18:00-22:00) [12]. This may mask the effect occurring at each time-period falling within such time window. The findings from this study are however consistent with reports on An. gambiae and An. funestus

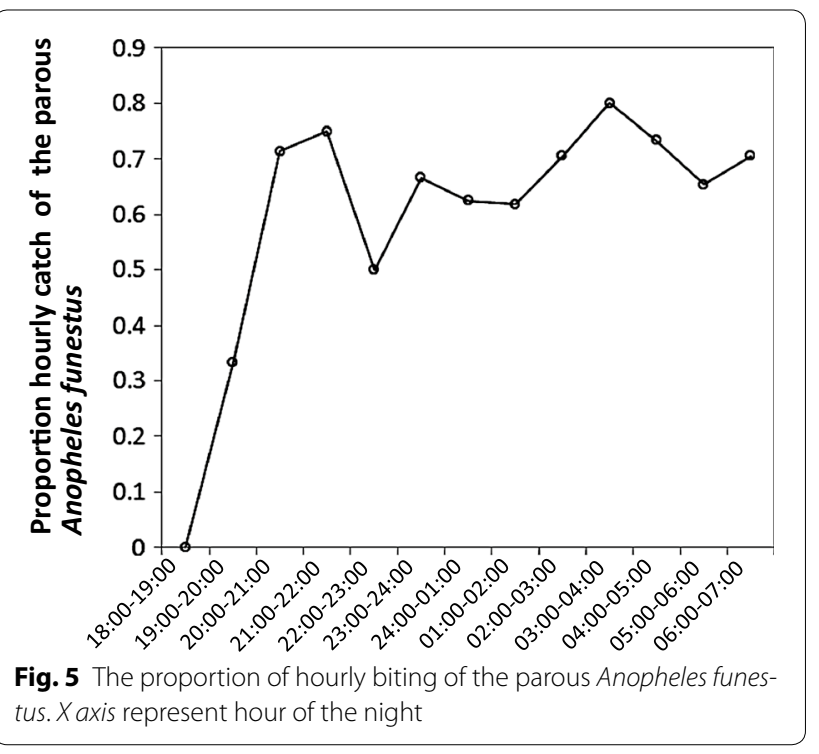

in Burkina Faso [54] and An. funestus in Tanzania [11]. These findings also support the conclusive statement by Gillies that opposed the idea that age of An. gambiae was an important characteristic in determining biting time. Although in his study he found on average more young mosquitoes biting in the early part of the night than in the middle hours (22:00-02:00), these observations were found to vary as a function of weeks. For instance, the proportion of young mosquito biting was highest in the middle hours of the night in 4 weeks out of an 8 -week study [13]. The absence of clustering of parous mosquitoes at specific time periods of the night may imply that the risk of human exposure to potentially infectious bites [6] is equally distributed throughout the biting window of these vectors. This also implies that protection against bites from these mosquitoes at all times is key to preventing malaria transmission. These suggestions may be supported by a recent epidemiological study in urban Dar es Salaam, Tanzania, which demonstrated that people who sleep inside houses with complete window screening and under a bed net enjoyed a reduction in infection risk only if their evenings and mornings were also spent indoors [55]. Time spent outdoors in the evenings and time of leaving the house in the mornings rather than living in a quality house alone [56], appeared to matter significantly in determining human infection risk [55].

Host-seeking $A n$. arabiensis and An. funestus were observed actively feeding at times when most local people are usually outdoors engaged in different activities. Most outdoor activities in Valley of Kilombero occur on average before 22.00 and after 05.00 [29]. This overlap in time and space between mosquito and human activities increases the risk of human exposure to mosquito bites outdoors and consequently infection transmission [55]. 


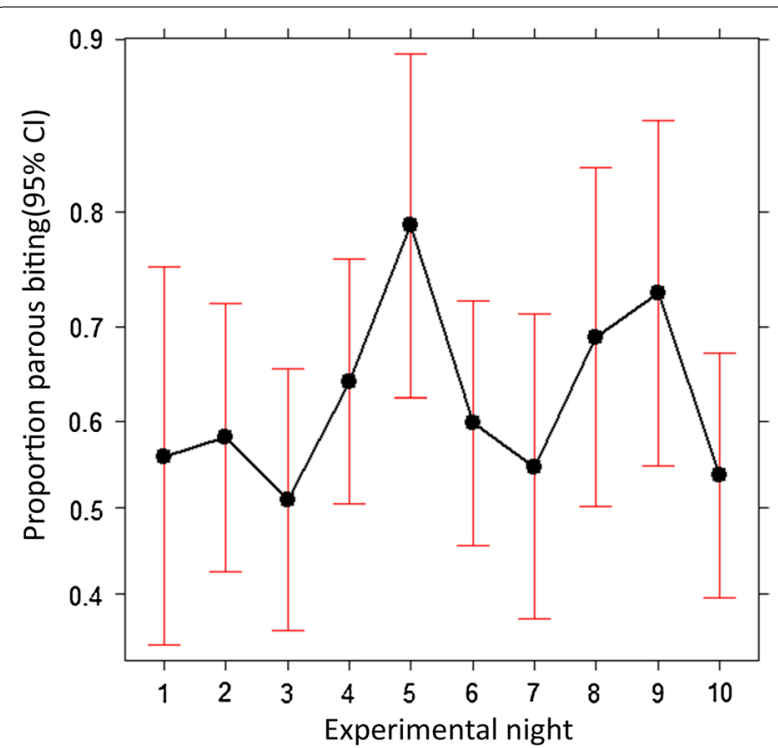

Fig. 6 Distribution of the proportion of parous Anopheles arabiensis that were recaptured biting at each night of the study in the semifield system. Data points represent absolute proportion of parous biting at each night of the study and Bars represent the 95\% confidence interval. Xaxis represents night of the study

This early evening biting peak of $A n$. arabiensis is consistent with a previous report from the same valley [29]. It is also consistent with results from other parts of Tanzania [57] and beyond [22]. The early morning peak biting by An. funestus may be reported for the first time in this setting, however, similar observations have been reported elsewhere, including recent reports from West Africa $[25,58]$. Early evening and morning outdoor exposure of humans to mosquito bites has epidemiological importance in terms of controlling transmission in this setting, and possibly across sub-Saharan Africa and beyond $[24,59,60]$ where ITNs and/or indoor residual spraying (IRS) remain the only interventions. New interventions should focus on disrupting malaria transmission beyond bedtime hours, specifically before and immediately after bedtime. Interventions such as insecticide-treated clothing, topical and spatial repellents [61-63], and the application of ivermectin [64] should be trialled.

In the SFS, more parous were recaptured compared to nulliparous. Whether this implies parous mosquitoes to be more active and aggressive in feeding than nulliparous counterpart remains unclear. The few existing studies, for instance on Aedes albopitus, although demonstrating variations in feeding responsiveness between parous and nulliparous females, such responsiveness was found to vary with time, and so was time dependent [65]. There could be many factors that affect this. It may also be true that parity effect of feeding propensity is species-specific; more work is needed to confirm this. It is also not clear whether the sugar-feed colour marking approach affected the propensity of these mosquitoes to feed; the low recapture rate of $49 \%$ is unsurprising in the SFS which contains vegetation (Lwetoijera et al. unpublished).

The biting time phenotype outcome observed with wild population of An. arabiensis in the field and in semifield-reared $A n$. arabiensis in the SFS was assessed to see if it compared well and thus genotype. The two mosquito populations, although of the same taxon and originated from the same valley and subject to variable environments, exhibited different biting tendencies. An. arabiensis in the SFS was observed to respond to and started biting heavily immediately upon introduction of a human host in the semi-field in the evening and dropped to zero as the night progressed. To the contrary, the biting pattern of wild $A n$. arabiensis in the full field was fairly distributed throughout the night (Fig. 3). This difference could clearly be due to the differences in environmental conditions they were exposed to. Although a search for a blood meal is mainly triggered by a physiological process in the mosquito, to locate, respond and successfully attack a host can be influenced by a number of environmental factors. These factors may include the distance between the host and the breeding site and the availability and accessibility of the host. Response to and successfully finding and attacking a host are the factors that are being measured in the field when characterizing behavioural phenotype outcomes of mosquito biting patterns $[24,25,59,66]$. Therefore, the observed increasing early and outdoor biting behaviour by malaria vectors across sub-Saharan Africa and beyond $[24,25,59,66]$ may be described as driven mainly by phenotypic plasticity in response to variable environments, rather than to genetic change $[67,68]$.

This experimental design for addressing whether biting activity patterns observed in the field are influenced by genetic change or simply phenotypic plasticity of preexisting behaviour had some limitations. Both field and SFS trials were supposed to be conducted in parallel on the same night and ideally with the SFS build at the vicinity of field collection so that the environmental variables between nights, such as temperature, humidity winds, moon light and cloud cover could also be controlled [69, 70]. Despite these limitations, this variation in behavioural outcomes observed between field and the SFS gives some insight into the role of availability and accessibility of host in determining biting time phenotypes but does not fully explain the observed field biting activity.

\section{Conclusion}

The study has shown that mosquito biting time phenotype is not influenced by parity status. These findings imply that the risk of human exposure to potentially infectious bites is equally distributed throughout the 
night. The peak biting activity by $A n$. arabiensis and $A n$. funestus which is outside normal hours when people could access and use bed nets, calls for optimization of vector control approaches.

\section{Authors' contributions}

NJG conceived and designed the experiments, performed the experiments, analysed the data, prepared the Figures, wrote the paper and reviewed drafts of the paper; MPM performed the experiments; MPM and MTS reviewed the manuscript. All authors read and approved the final manuscript.

\section{Author details}

1 Ifakara Health Institute, Environmental Health and Ecological Sciences Thematic Group, Coordination Office, PO Box 78373, Kiko Avenue, Mikocheni, Dar es Salaam, United Republic of Tanzania. ${ }^{2}$ Marquette University, Department of Mathematics, Statistics and Computer Sciences, Milwaukee, WI, USA. ${ }^{3}$ QIMR Berghofer Medical Research Institute, Herston, QLD 4006, Australia.

\section{Acknowledgements}

We thank the residents of Minepa Village for their cooperation throughout the study and to the volunteers for their commitment to this work. Special thanks go to Japhet Kihonda for doing a wonderful job with dissection of mosquitoes collected.

\section{Competing interests}

The authors declare that they have no competing interests.

\section{Availability of data and materials}

Access and use of data supporting this article will have to comply with the Ifakara Health Institute data sharing policy. If data are requested and no competing interest is apparent, the requested data will be made available under defined conditions expressed in writing through an exchange of letters between parties stipulating those conditions and any agreed limits to use of data.

\section{Consent for publication}

Written informed consent was obtained from volunteers for participation in the study and for publication of this report and any accompanying images. Consent and approval for publication was also obtained from the Medical Research Coordination Committee of the National Institute of Medical Research in Tanzania.

\section{Ethical approval and consent to participate}

Research clearance was obtained from the institutional review board of Ifakara Health Institute in Tanzania (IHI/IRB/No. 16-2014), and Medical Research Coordination Committee of the National Institute of Medical Research in Tanzania (NIMR/HQ/R.8a/Vol.IX/1925). All participants conducting HLCs were provided with drug prophylaxis (Malarone ${ }^{\circledR}$ ) against malaria [49] and were screened for malaria parasites by rapid diagnostic test (mRDT (MAL-Pf ${ }^{\circledR}$, ICT Diagnostics, Cape Town, South Africa, which detects histidine-rich protein II) before and after the study. Before implementation of the study, written informed consent was obtained from the volunteers and heads of household for their participation.

\section{Funding}

This work was supported by the Wellcome Trust (Research Training Fellowship for Public Health and Tropical Medicine number 102368/Z/13/Z) awarded to NJG

Received: 15 September 2016 Accepted: 20 February 2017

Published online: 28 February 2017

\section{References}

1. Gillies MT, DeMeillon B. The Anophelinae of Africa south of the Sahara (Ethiopian zoogeographical region). Johannesburg: South African Institute for Medical Research; 1968.

2. Gillies MT. Anopheline mosquitos: vector behaviour and bionomics. Edinburgh: Churchill Livingstone; 1988.
3. MacDonald G. The epidemiology and control of malaria. London: Oxford University Press; 1957.

4. Garrett-Jones C. Prognosis for interruption of malaria transmission through assessment of the mosquito's vectorial capacity. Nature. 1964;204:1173-5.

5. Beier JC. Malaria parasite development in mosquitoes. Ann Rev Entomol. 1998:43:519-43.

6. Smith DL, Dushoff J, McKenzie FE. The risk of a mosquito-borne infection in a heterogeneous environment. PLoS Biol. 2004;2:e368.

7. Dye $C$. The analysis of parasite transmission by bloodsucking insects. Ann Rev Entomol. 1992:37:1-19.

8. Gillies MT, Wilkes TJ. A study of the age-composition of populations of Anopheles gambiae Giles and A. funestus Giles in North-Eastern Tanzania. Bull Entomol Res. 1965;56:237-62.

9. Charlwood JD, Wilkes TJ. Studies on the age composition of sample of Anopheles darlingi Root (Diptera: Culicidae) in Brazil. Bull Entomol Res. 1979;69:337-42.

10. Charlwood JD, Paru R, Dagoro H, Lagog M. Influence of moonlight and gonotrophic age on biting activity of Anopheles farauti (Diptera: Culicidae) from Papua New Guinea. J Med Entomol. 1986;23:132-5.

11. Gillies MT, Wilkes TJ. Observations on nulliparous and parous rates in populations of Anopheles funestus in East Africa. Ann Trop Med Parasitol. 1963;57:204-13.

12. Bockarie MJ, Alexander N, Bockarie F, Ibam E, Barnish G, Alpers M. The late biting habit of parous Anopheles mosquitoes and pre-bed exposure of humans to infective female mosquitoes. Trans R Soc Trop Med Hyg. 1996;90:23-5.

13. Gillies MT. Age grouping and the biting cycle in Anopheles gambiae. A preliminary investigation. Bull Entomol Res. 1957;48:553-9.

14. Lengeler $C$. Insecticide-treated bed nets and curtains for preventing malaria. Cochrane Database Syst Rev. 2004;363:CD000363.

15. WHO. World Malaria Report 2014. Geneva: World Health Organization; 2014.

16. O'Meara WP, Bejon P, Mwangi TW, Okiro EA, Peshu N, Snow RW, et al. Effect of a fall in malaria transmission on morbidity and mortality in Kilifi, Kenya. Lancet. 2008;372:1555-62.

17. Battarai A, Ali AS, Kachur SP, Martensson A, Abbas AK, Khatib R, et al. Impact of artemisinin-based combination therapy and insecticidetreated nets on malaria burden in Zanzibar. PLoS Med. 2007;4:e309.

18. Murray CJL, Rosenfeld LC, Lim SS, Andrews KG, Foreman KJ, Haring D, et al. Global malaria mortality between 1980 and 2010: a systematic analysis. Lancet. 2012;379:413-31.

19. Bhatt S, Weiss DJ, Cameron E, Bisanzio D, Mappin B, Dalrymple U, et al. The effect of malaria control on Plasmodium falciparum in Africa between 2000 and 2015. Nature. 2015;526:207-11.

20. Bayoh MN, Mathias DK, Odiere MR, Mutuku FM, Kamau L, Gimnig JE, et al. Anopheles gambiae: historical population decline associated with regiona distribution of insecticide-treated bed nets in Western Nyanza Province, Kenya. Malar J. 2010;9:62.

21. Russell TL, Govella NJ, Azizi S, Drakeley CJ, Kachur SP, Killeen GF. Increased proportions of outdoor feeding among residual malaria vector populations following increased use of insecticide-treated nets in rural Tanzania. Malar J. 2011;10:80

22. Yohannes M, Boelee E. Early biting rhythm in the Afro-tropical vector of malaria, Anopheles arabiensis and challenges for its control in Ethiopia. Med Vet Entomol. 2012;26:103-5.

23. Reddy MR, Overgaard HJ, Abaga S, Reddy VP, Caccone A, Kiszewski AE, et al. Outdoor host seeking behaviour of Anopheles gambiae mosquitoes following initiation of malaria vector control on Bioko Island, Equatorial Guinea. Malar J. 2011;10:184.

24. Moiroux N, Gomez MB, Pennetier C, Elanga E, Djenontin A, Chandre F, et al. Changes in Anopheles funestus biting behavior following universal coverage of long-lasting insecticidal nets in Benin. J Infect Dis. 2012;206:1622-9.

25. Sougoufara S, Diedhiou SM, Doucoure S, Diagne N, Sembene PM, Harry $\mathrm{M}$, et al. Biting by Anopheles funestus in broad daytime after use of long-lasting insecticidal nets: a new challenge to malaria control. Malar J. 2014;13:125.

26. Mwangangi JM, Mbogo CM, Orindi BO, Muturi EJ, Midega JT, Nzovu $J$, et al. Shifts in malaria vector species composition and transmission dynamics along the Kenyan coast over the past 20 years. Malar J. 2013;12:13. 
27. Ndiath MO, Mazenot C, Sohna C, Trape J-F. How the malaria vector Anopheles gambiae adapts to the use of insecticide-treated nets by African population. PLoS ONE. 2014;9:e97700.

28. Lwetoijera DW, Harris C, Kiware SS, Dongus S, Devine GJ, McCall PJ, et al. Increasing role of Anopheles funestus and Anopheles arabiensis in malaria transmission in the Kilombero Valley, Tanzania. Malar J. 2014;13:331.

29. Matowo NS, Moore J, Mapua S, Madumla EP, Moshi IR, Kaindoa EW, et al. Using a new odour-bated device to explore options for luring and killing outdoor-biting malaria vectors: a report on design and field evaluation of the Mosquito Landing Box. Parasit Vectors. 2013;6:137.

30. NBS. Tanzania HIV/AIDS and Malaria Indicator Survey 2011-2012. Tanzania: National Bureau of Statistics; 2013. p. 1-229.

31. Killeen GF, Tami A, Kihonda J, Okumu FO, Kotas ME, Grundmann H, et al. Cost-sharing strategies combining targeted public subsidies with privatesector delivery achieve high bednet coverage and reduced malaria transmission in Kilombero valley, southern Tanzania. BMC Infect Dis. 2007;7:121.

32. Kreppel K, Johnson PCD, Govella NJ, Pombi M, Maliti DF, Ferguson H. Comparative evaluation of the Sticky-Resting-Box-Trap, the standardised resting-bucket-trap and indoor aspiration for sampling malaria vectors. Parasit Vectors. 2015;8:462.

33. Russell TL, Lwetoijera DW, Maliti D, Chipwaza B, kihonda J, Charlwood JD, et al. Impact of promoting longer-lasting insecticide treatment of bed nets upon malaria transmission in a rural Tanzanian setting with preexisting high coverage of untreated nets. Malar J. 2010;9:187.

34. NBS. Tanzania HIV/AID malaria indicator survey 2007-2008. Dar es Salaam: National Bureau of Statistics; 2008. p. 1-127.

35. Mboera LEG. Sampling techniques for adult Afrotropical malaria vectors and their reliability in the estimation of entomological inoculation rates. Tanzan Health Res Bull. 2005;7:117-24.

36. Service MW. Mosquito ecology: field sampling methods. 2nd ed. London: Elsevier applied Science; 1993.

37. Gillies MT, Coetzee M. A supplement to the Anophelinae of Africa South of the Sahara (Afrotropical region). Johannesburg: South African Medical Research Institute; 1987.

38. Detinova TS. Age-grouping methods in Diptera of medical importance, with special reference to some vectors of malaria. Geneva: World Health Organization; 1962.

39. Scott JA, Brogdon WG, Collins FH. Identification of single specimens of Anopheles gambiae complex by polymerase chain reaction. Am J Trop Med Hyg. 1993;49:520-9.

40. Wirtz RA, Duncan JF, Njelesani EK, Schneider I, Brown AE, Oster CN, et al. ELISA method for detecting Plasmodium falciparum circumsporozoite antibody. Bull World Health Organ. 1989;67:535-42.

41. Burkot TR, Graves PM, Cattani JA, Wirtz RW, Gibson FD. The efficiency of sporozoite transmission in the human malarias, Plasmodium falciparum and P. vivax. Bull World Health Organ. 1987;65:375-80.

42. Durnez L, Van Bortel W, Denis L, Roelants P, Veracx A, Trung HD, et al. False positive circumsporozoite protein ELISA: a challenge for the estimation of the entomological inoculation rate of malaria and for vector elimination. Malar J. 2011;10:195.

43. Ferguson HM, Ng'habi KR, Walder T, Kadungula D, Moore SJ, Lyimo I, et al. Establishment of a large semi-field system for experimental study of African malaria vector ecology and control in Tanzania. Malar J. 2008;7:158.

44. Ng'habi KR, Mwasheshi D, Knols BG, Ferguson HM. Establishment of a self-propagating population of the African malaria vector Anopheles arabiensis under semi-field conditions. Malar J. 2010;9:356.

45. Mascari TM, Foil LD. Laboratory evaluation of the efficacy of fluorescent biomarkers for sugar-feeding sand flies (Diptera: Psychodidae). J Med Entomol. 2010;47:664-9.

46. Stewart ZP, Oxborough RM, Tungu PK, Kirby MJ, Rowland MW, Irish SR Indoor application of attractive toxic sugar bait (ATSB) in combination with mosquito nets for control of pyrethroid-resistant mosquitoes. PLoS ONE. 2013;8:e84168.

47. Charlwood JD, Graves PM, Birley MH. Capture-recapture studies with mosquitoes of the group Anopheles punctulatus Donitz (Diptera: Culicidae) from Papua New Guinea. Bull Entomol Res. 1986;76:211-27.

48. Achee NL, Grieco JP, Andre RG, Rejmankova E, Roberts DR. A mark-release -recapture study to define the flight behaviors of Anopheles vestitipennis and Anopheles albimanus in Belize, Central America. J Am Mosq Control Assoc. 2007;23:276-82.
49. Gimnig JE, Walker ED, Otieno P, Kosgei J, Olang G, Omboki M, et al. Incidence of malaria among mosquito collectors conducting human landing catches in western Kenya. Am J Trop Med Hyg. 2013;88:301-8.

50. Lindsay S, Ansell J, Selman C, CoxV, Hamilton K, Walraven G. Effect of pregnancy on exposure to malaria mosquitoes. Lancet. 2000;355:1972.

51. Omolo MO, Njiru B, Ndiege IO, Musau RM, Hassannali A. Differential attractiveness of human foot odours to Anopheles gambiae Giles sensu stricto (Diptera: Culicidae) and variation in their chemical composition. Acta Trop. 2013;128:144-8.

52. Lindsay SW, Adiamah JH, Miller JE, Pleass RJ, Armstrong JRM. Variation in the attractiveness of human subjects to malaria mosquitoes (Diptera: Culicidae) in The Gambia. J Med Entomol. 1993;30:368-73.

53. Cook MK, Kahindi SC, Oriango RM, Owaga C, Ayoma E, Mabuka D, et al. A bite before bed': exposure to malaria vectors outside the times of net use in the highlands of western Kenya. Malar J. 2015;14:259.

54. Maxwell CA, Wakibara J, Tho S, Curtis CF. Malaria-infective biting at different hours of the night. Med Vet Entomol. 1998;12:325-7.

55. Msellemu D, Namango HI, Mwakalinga VM, Ntamatungiro AJ, Mlacha Y, Mtema ZJ, et al. The epidemiology of residual Plasmodium falciparum malaria transmission and infection burden in an African city with high coverage of multiple vector control measures. Malar J. 2016;15:288.

56. Tusting LS, Ippolito MM, Willey BA, Kleinschmidt I, Dorsey G, Gosling RD, et al. The evidence for improving housing to reduce malaria: a systematic review and meta-analysis. Malar J. 2015;14:209.

57. Govella NJ, Okumu FO, Killeen GF. Insecticide-treated nets can reduce malaria transmission by mosquitoes which feed outdoors. Am J Trop Med Hyg. 2010;82:415-9.

58. Moiroux N, Damien GB, Egrot M, Djenontin A, Chandre F, Corbel V, et al. Human exposure to early morning Anopheles funestus biting behavior and personal protection provided by long-lasting insecticidal nets. PLOS ONE. 2014;9:e104967.

59. Killeen GF. Characterizing, controlling and eliminating residual malaria transmission. Malar J. 2014;13:330.

60. Russell TL, Beebe NW, Cooper RD, Lobo NF, Burkot TR. Successful malaria elimination strategies require interventions that target changing vector behaviours. Malar J. 2013;12:56.

61. Wilson AL, Chen-Hussey V, Logan JG, Lindsay SW. Are topical insect repellents effective against malaria in endemic populations? A systematic review and meta-analysis. Malar J. 2014;13:446.

62. Maia MF, Onyango SP, Thele M, Simfukwe E, Turner E, Moore SJ. Do topical repellents divert mosquitoes within a community? Health equity implications of topical repellents as a mosquito bite prevention tool. PLoS ONE. 2013;8:e84875

63. Govella NJ, Ogoma SB, Paliga J, Chaki PP, Killeen GF. Impregnating hessian strips with the volatile pyrethroid transfluthrin prevents outdoor exposure to vectors of malaria and lymphatic filariasis in urban Dar es Salaam. Parasit Vectors. 2015:8:322.

64. Chaccour CJ, Rabinovich NR, Slater H, Canavati SE, Bousema T, Lacerda $M$, et al. Establishment of the Ivermectin research for malaria elimination network: updating the research agenda. Malar J. 2015;14:243.

65. Xue RD, Barnard DR. Human host avidity in Aedes albopictus. influence of mosquito body size, age, parity, and time of day. J Am Mosq Control Assoc. 1996;12:58-63.

66. Durnez L, Mao S, Denis L, Roelants P, Sochantha T, Coosemans M. Outdoor malaria transmission in forested villages of Cambodia. Malar J. 2013;12:329.

67. Govella NJ, Chaki PP, Killeen GF. Entomological surveillance of behavioural resilience and resistance in residual malaria vector populations. Malar J. 2013;12:124.

68. Maliti DF, Marsden CD, Main BJ, Govella NJ, Yamasaki Y, Collier TC, et al. Investigating associations between biting time in the malaria vector Anopheles arabiensis Patton and single nucleotidepolymorphisms in circadian clock genes: support for sub-structure among An. arabiensis in the Kilombero valley of Tanzania. Parasit Vectors. 2016;9:109.

69. Kampango A, Cuamba N, Charlwood JD. Does moonlight influence the biting behaviour of Anopheles funestus. Med Vet Entomol. 2011;25:240-6.

70. Davies JB. Moonlight and the biting activity of Culex (Melanoconion) portesi Senevet \& Abonnenc aud C. (M.) taeniopus D. \& K. (Diptera, Culicidae) in Trinidad forests. Bull Entomol Res. 1975;65:81-96. 